given a course of "deep X-ray therapy" for some weeks during convalescence and after.

In the present state of our knowledge I feel that it is doubtful policy to rely too much on any one method of treatment for these cases, but that we should remove the growth as cleanly and as widely as possible, and then subject the site of operation to radio-activity in order to stamp out any elements of cancer which may remain. Finally, we must pay every attention and concentrate our efforts towards improving technique and correlating results.

With regard to the results of treatment of malignant disease of the prostate and bladder, we must admit that the outlook is still far from bright and that a large number of these people do not survive more than a year or so after the onset of symptoms. The great difficulty is the insidious nature of the onset of the trouble, which makes it essential that we should be on the outlook for early symptoms, so that we may be able to proceed with more hope of a successful issue, and not, as is too often the case, meet our patient when he is beyond hope of anything but the possibility of making him comfortable and perhaps of adding a few months to his existence.

Little remains; we have in surgery and radiation treatment two powerful weapons which, if given a chance, will help us greatly, but too much must not be expected of them at present. The disease is a desperate one and the results of treatment are still imperfect. There has, however, been some improvement, and it is encouraging to find that without doubt in many cases we have a method which will often allay symptoms, cause shrinkage of the growth, and prolong life.

\section{OBSTRUCTION OF THE COLON.}

\author{
$B y$ W. B. GABRIEL,
}

M.S., F.R.C.S.,

Surgeon to the Royal Northern Hospital, Senior Assistant Surgeon to St. Mark's Hospital for Diseases of the Rectum.

IT is the common experience among surgeons that resection of the colon is a tricky and dangerous operation. The factors which contribute to the high mortality of resection and anastomosis of the colon are as follows :-

(I) The patients are often advanced in age, with poor healing powers.

(2) A state of subacute obstruction often exists which it is difficult to overcome even after careful bowel preparation.

(3) The contents of the colon are extremely infective and the risk of peritonitis is much greater than after resection of any other part of the gastro-intestinal tract.

(4) The technical difficulties in rnaking thoroughly sound anastomosis, whether lateral or end-to-end, are often very great; there may be great disproportion in size between the bowel lumen above and below the site of obstruction; atrophy or hypertrophy of the bowel wall may make suturing difficult, and the overloading with fat and enlarged appendices epiploicæ which is so commonly met with make it difficult to insert a uniformly firm line of peritoneal sutures. The blood supply to the upper and lower ends has to be carefully considered on anatomical lines if a $\mathrm{V}$-shaped portion of meso-colon is to be excised, and excessive zeal in placing sutures has to be avoided for fear of damaging the vascularity of the anastomosis. Even if a cæcostomy is done at the conclusion of the operation the risk of leakage at the junction is not entirely prevented. Other points calling for anxious consideration by the surgeon are whether local drainage should be arranged for at the side of the anastomosis and the decision as 
to when graded aperients can safely be administered.

For these many reasons there is at the present time a tendency in suitable cases to revert to the old operation originally described by Pdul [I] in I895, also known as the Mikulicz operation. Sistrunk [2] of the Mayo Clinic has recently written at some length on the possibilities of the operation, and Rankin [3] has described a modification of the operation, using a special double crushing clamp, which he has called "obstructive resection." Devine [4] has also described another modification which has the object of shortening and facilitating the final stage of closure.

The principle of the operation, when applied to resecting a carcinoma of the colon, is to exteriorize the affected portion of bowel, to resect the required amount of bowel and adjacent meso-colon, and arrange side by side a double-barrelled colostomy; at a later stage the colostomy spur is crushed by a suitable enterotome, and finally an extraperitoneal closure of the fæcal fistula is performed. Several important details of the final operation and the preparation for it, as described by Greig-Smith, were published by Cawardine [5] in 1926 ; this article well repays careful stıdy.

The following recent case of acute intestinal obstruction due to a chronic intussusception of the colon may now be described; it is an example of the value of the multiplestage operation.

Case.-The patient is a woman, aged 68 ; she gave a history of increasing constipation with attacks of abdominal pain for twelve months; no tumour could be felt on rectal examination, and she was admitted into hospital with a diagnosis of probable carcinoma of the colon. A few days later her symptoms became more acute: the abdomen became distended and enemata produced no results except some mucus and foul-smelling discharge.

First Operation, October 2, 1930.-The abdomen was now greatly distended but not sufficiently so to prohibit a quick laparotomy. Under general anæsthesia I explored the abdomen through a right paramedian incision; the large bowel was enormously distended and with a hand in the pelvis I could feel a soft movable mass in the lower pelvic colon, the nature of which I could not then determine. Through a separate incision in the right iliac region I was able to bring out an adequate portion of the distended cæcum and by means of a Taylor's clamp an " ink-bottle" cæcostomy was performed; the tube inserted was a flanged one prepared by cutting off the conical end of a large-sized de Pezzer catheter, No. 32 French scale. The abdominal distension was immediately relieved and closing the main incision was simple. A few ounces of saline solution with glucose were run through the cæcostomy tube every two hours, with the object both of supplying the necessary fluid and also to ensure that the tube remained patent. The patient's general condition was satisfactory and four days later I considered that the critical time had come for the attempt to decide on and if possible to remove the cause of the obstruction.

Second Operation, October I3, I930.Laparotomy was performed, under general anæsthesia, through a left paramedian incision. The patient was placed in a full Trendelenburg position and coils of intestine packed out of the pelvis. The pelvic mesocolon was found to be abnormally short and then it was apparent that an intussusception of the pelvic colon had occurred; the point where the entering layer disappeared into the ensheathing layer was easily demonstrated and the distended bowel occupied by the intussuscepted part could be felt reaching down below the peritoneal reflection. Gentle traction produced very little effect, so my house surgeon passed two fingers into the patient's rectum and could feel the apex of the intussusception clearly; by a combination of taxis on his part and traction and squeezing on mine the 
intussusception was safely reduced. A long pelvic loop was then available, with a soft tumour palpable in the middle of the pelvic colon at the point that had formed the apex of the intussusception. The tumour was not clearly pedunculated and in view of the patient's age and history I decided to treat it as a carcinoma. A separate incision was made in the left iliac region and the pelvic loop brought out. After closing and packing of the main incision, I excised the tumour and neighbouring portion of meso-
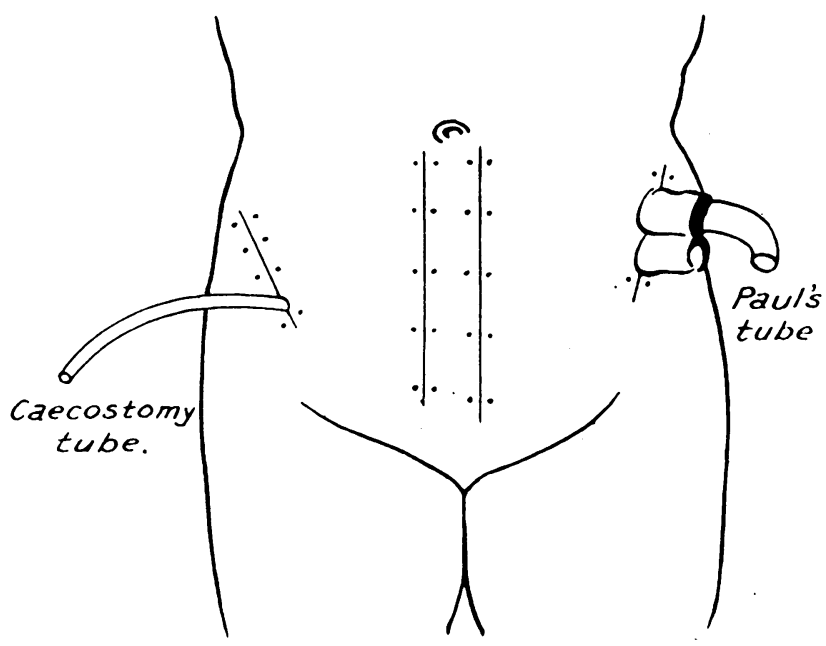

Diagram showing operative procedures in this case.

First operation (right side), laparotomy and cæcostomy. Second operation (left side), laparotomy and resection of colon by Paul's method.

colon. The afferent and efferent loops were sutured together on the outer aspect for a distance of 2 in. and a Paul's tube was tied into the proximal opening.

Present State.-The patient's course since the second operation has been uneventful; her temperature and pulse are normal ; she takes light diet and feels well. She has a double-barrelled colostomy which is now well adherent to the abdominal wall. The cæcostomy tube is still in situ, there has been no leakage beside it and the daily colon wash-outs are being given through it.
I propose, in a few days' time, to apply a Dupuytren's enterotome to crush the spur $\stackrel{\mathbb{Q}}{\Omega}$ and the usual extraperitoneal closure will.. follow a few weeks later.

The specimen removed measured 5 in. in 0 length. It showed a soft ulcerated and sessile tumour, oval in shape, on which my $\frac{\vec{\sigma}}{\overrightarrow{0}}$ colleague, Dr. Dukes, reports as follows: "Microscopic examination shows the is tumour to be an adenocarcinoma which $\overrightarrow{0}$ has a villous character on the surface and $\vec{\omega}$ the structure of an adenocarcinoma in the region where infiltration is taking place. Spread by direct continuity has taken place as far as the longitudinal muscle coat, and o the tumour will be classified as a $\mathrm{B} 2$ case. i The general appearances are those of ao carcinoma which has developed in a preexisting adenoma." The prognosis as $z$ regards recurrence is therefore good.

\section{SUMMARY.}

The method adopted in this case hos $\overrightarrow{0}$ allowed of relief of an acute intestint? obstruction and adequate removal of carcinoma of the colon in stages. More-s over, the mobile pelvic colon will finally have such anchorage by adhesions to the deep aspect of the anterior abdominal wall $\stackrel{?}{\longrightarrow}$ that recurrence of the intussusception will be very improbable.

\section{REFERENCES.}

[1] PAUL, F. T. Liverpool Med. and Chirurg. Journ. 1895.

[2] Sistrunk, Walter E. Annals of Surg., 1928, lxxxviii, 597.

[3] Rankin, F. W. "Obstructive Resection of theo Colon," Surg. Gyn. and Obst., 1930, 1, 594.

[4] Devine, H. B. "Colon Surgery in the Debilio tated," Journ. of the College of Surgeons of Australasia, November, 1928, 173.

[5] Cawardine, T. Brit. Journ. Surg., xiv, No. 54 N 329. 